\title{
Management of primary ciliary dyskinesia in European children: recommendations and clinical practice
}

\author{
Marie-Pierre F. Strippoli, Thomas Frischer, Angelo Barbato, Deborah Snijders, \\ Elisabeth Maurer, Jane S.A. Lucas, Ernst Eber, Bulent Karadag, Petr Pohunek, \\ Zorica Zivkovic, Amparo Escribano, Chris O'Callaghan, Andrew Bush and Claudia \\ E. Kuehni, for the ERS Task Force on primary ciliary dyskinesia in children
}

ABSTRACT: The European Respiratory Society Task Force on primary ciliary dyskinesia (PCD) in children recently published recommendations for diagnosis and management. This paper compares these recommendations with current clinical practice in Europe.

Questionnaires were returned by 194 paediatric respiratory centres caring for PCD patients in 26 countries. In most countries, PCD care was not centralised, with a median (interquartile range) of 4 (2-9) patients treated per centre. Overall, $90 \%$ of centres had access to nasal or bronchial mucosal biopsy. Samples were analysed by electron microscopy $(77 \%)$ and ciliary function tests (57\%). Nasal nitric oxide was used for screening in $46 \%$ of centres and saccharine tests in $36 \%$. Treatment approaches varied widely, both within and between countries. European region, size of centre and the country's general government expenditure on health partly defined availability of advanced diagnostic tests and choice of treatments.

In conclusion, we found substantial heterogeneity in management of PCD within and between countries, and poor concordance with current recommendations. This demonstrates how essential it is to standardise management and decrease inequality between countries. Our results also demonstrate the urgent need for research: to simplify PCD diagnosis, to understand the natural history and to test the effectiveness of interventions.

KEYWORDS: Bronchiectasis, ciliary motility disorders, diagnosis, Kartagener syndrome, primary ciliary dyskinesia, therapy

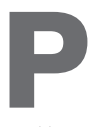

rimary ciliary dyskinesia $(\mathrm{PCD})$ is a rare autosomal recessive disease, characterised by altered ciliary beat frequency or beat pattern or both. It results in an impaired mucociliary clearance of upper and lower airways, leading to chronic airway inflammation and infection [1-6]. Published data on PCD are scarce, observational and derived from small case series.

The European Respiratory Society (ERS) Task Force on PCD in children was founded in 2006 with the aims of describing diagnostic and therapeutic approaches for this rare disease in Europe, collecting cross-sectional and longitudinal data on representative numbers of patients, defining research needs, and enhancing collaborative research. A consensus statement published in 2009 summarises recommended diagnostic and treatment approaches based on available scientific evidence and clinical expertise of the task force members [7]. The consensus statement concluded that the evidence base for diagnosis and treatment of PCD is poor and more research urgently needed. The Task Force also made a number of recommendations (table 1). Although these partly reflect expert opinion rather than scientific evidence, they reflect the best currently available evidence.

It is unclear how these recommendations compare to current clinical practice in Europe. For this reason, the PCD Task Force sent a questionnaire to all paediatric respiratory centres in Europe. It aimed to describe: 1) how care for children with PCD is organised (centralised versus split); 2) how these centres screen for PCD and how they confirm the diagnosis; 3) which treatment options are used; and 4) how
AFFLLIATIONS

For affiliation details for all authors, and for a full list of Task Torce members, please refer to the Acknowledgements section.

CORRESPONDENCE

C.E. Kuehni

Institute of Social and

Preventive Medicine

University of Bern

Finkenhubelweg 11

CH-3012 Bern

Switzerland

E-mail: kuehni@ispm.unibe.ch

Received:

May 022011

Accepted after revision:

Aug 122011

First published online:

Jan 262012 


\section{TABLE 1 Recommendations for diagnosis and management of primary ciliary dyskinesia (PCD)}
A) Centralised care
1) Patients with PCD should be seen for either full or shared care in a centre specialising in the condition

\section{B) Diagnosis}

2) Nasal NO levels can be used as a screening test for PCD in children, while the saccharine test is unreliable

3) Diagnosis is confirmed by analysis of ciliated epithelial cells derived from nasal brushings or bronchoscopic samples

4) Ciliary beat pattern and frequency analyses using high-speed video recording and electron microscopy are the key diagnostic techniques; other techniques (cell culture, analysis of dynein protein localisation by immunofluorescence and genetic analyses) might help in selected patients

\section{C) Treatment}

5) Airway clearance by physiotherapy and exercise, and prompt antibiotic treatment (oral, intravenous if needed) are the cornerstones of treatment

6) Prophylactic oral antibiotics and long-term use of nebulised anti-Pseudomonas antibiotics should be considered in specific patients

7) Inhaled bronchodilators and topical or inhaled steroids have no routine place in PCD treatment except for patients with concurrent asthma; rhDNase and hypertonic saline might possibly be considered in very selected patients

8) The use of tympanostomic ventilation tubes should be avoided for PCD patients whenever possible

9) All PCD patients should receive all childhood immunisations including pneumococcal and influenza immunisation

D) Follow-up

10) A protocolised shared-care system is recommended to ensure specialist follow-up and prevent eventual lung damage; regular sputum or cough-swab cultures should be performed

NO: nitric oxide; rhDNase: recombinant human deoxyribonuclease. Summarised from the consensus statement of the European Respiratory Society Task Force on PCD in children [7]

long-term follow-up is organised. The underlying motivation was to detect regions within Europe and management topics where standardisation of procedures is particularly urgent. Conversely, recommendations that contrast with widespread clinical practice might need re-evaluation by randomised trials.

\section{METHODS}

\section{Study design and population}

Using a two-stage sampling design, we performed a questionnaire survey directed to all institutions considered likely to treat paediatric PCD patients in Europe. From the ERS membership roster, we found a national representative for most European countries. Turkey and Israel volunteered, and were also included. Questionnaires were distributed between January 2007 and October 2008, and replies collected until January 31, 2009, i.e. before publication of the Task Force consensus statement.

Each national representative made a list of all paediatric respiratory centres in his/her country and mailed the questionnaires. Non-responding centres were reminded repeatedly by letters, e-mails and personal phone calls. Depending on the national healthcare systems, national representatives sent questionnaires only to tertiary care centres (in countries with centralised care for paediatric PCD) or to tertiary, secondary and primary care centres (in countries where PCD care was not centralised).

\section{Definitions}

Type of centres was defined as tertiary care centres (university hospitals or tertiary referral centres), secondary care centres (regional referral centres with a respiratory unit) and primary care centres (paediatric practices or small hospitals).

Participating countries were grouped into five regions according to the United Nations definition of the European regions [8], with the following exceptions: UK and Ireland were analysed as a separate region, labelled the British Isles, given the large number of cases available from the UK; and Estonia, Israel, Serbia and Turkey were grouped with eastern Europe.

Data on general government expenditure on health (GGHE; the sum of outlays for health maintenance, restoration or enhancement paid for in cash or supplied in kind by government entities) were obtained from WHOSIS, the WHO Statistical Information System database bringing together core health statistics for the 193 World Health Organization member states [9].

\section{Questionnaire}

The 10-page questionnaire included a first section on centre characteristics and the team involved in PCD care and approximate numbers of PCD and cystic fibrosis patients cared for in the centre. Section two listed different diagnostic methods and asked whether these were currently available for diagnosing PCD, either locally at the centre, or upon referral to a regional or national reference centre. Section three listed potential treatments and asked if these were prescribed routinely (for all patients), frequently or sometimes (for some patients) or never. We also assessed the usual frequency of follow-up visits in the respective centre. Finally, the questionnaire included an anonymous list with characteristics of all PCD patients currently in follow-up. The questions used for this paper are listed in the online supplement; the full questionnaire is available from the authors on request. Results on individual patient data have been published [10].

\section{Analysis}

We double-entered all questionnaires into an EpiData database, eliminated double counts of cases reported by more than one centre, and analysed the data using Stata statistical software (version 10; STATA Corporation, College Station, TX, USA). To ensure comparability between countries, response rate was only computed for tertiary care centres.

For the analysis of reported practices for diagnostic work-up and management, we included only questionnaires from 


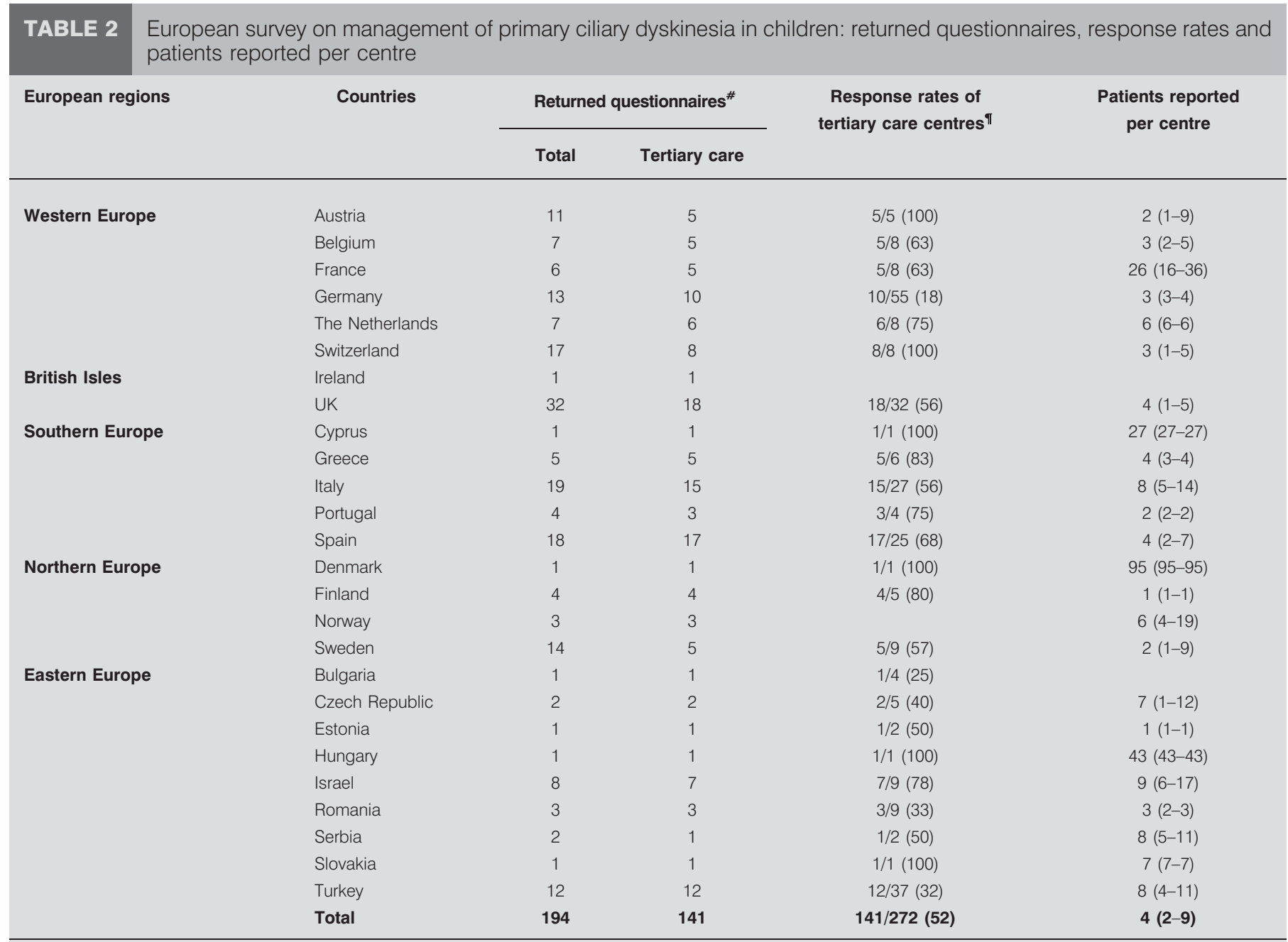

Data are presented as $\mathrm{n}, \mathrm{n} / \mathrm{N}(\%)$ or median (interquartile range). ${ }^{*}:$ all questionnaires that contained a list of patients; ${ }^{\circ}:$ calculated as the number of questionnaires returned by tertiary care paediatric centres divided by the total number of tertiary care paediatric centres in the respective country.

centres reporting cases. We used univariable and multivariable logistic regression models to determine factors associated with different diagnostic procedures and treatments. All factors associated with the outcome $(p<0.05)$ were retained in the multivariable model.

\section{RESULTS}

We received questionnaires from 223 centres. Of these, 194 centres (141 tertiary care and 53 smaller ones) in 26 countries reported PCD patients and were used for the analyses. The overall response rate from tertiary care centres was 52\%, ranging from $18 \%$ to $100 \%$ per country (table 2), with the majority of countries having a response rate of $>66 \%$.

\section{Organisation of care and number of patients per centre}

Three countries had a single national reference centre for PCD (Cyprus, Denmark and Hungary), while in other countries, PCD care was split among numerous centres (UK 32, Italy 19, Spain 18, Switzerland 17, Sweden 14 and Austria 11; table 2). The median number of patients per centre was 4 (interquartile range 2-9; range 1-95), with large differences between countries.

\section{Diagnosis of PCD}

The techniques available for PCD diagnosis in the five European regions, irrespective of whether the test was performed in the centre itself or whether patients were referred to a reference centre, are described in table 3 . In some countries (Bulgaria, Estonia, Hungary, Portugal and Romania), essential techniques were not available within the country (table E1 of the online supplement). Diagnostic techniques did not vary by type of centre, with the exception of electron microscopy and ciliary beat frequency and pattern, which were both more common in tertiary care (table E2 of the online supplement). The proportion of centres performing these measurements inhouse was lower for demanding investigations such as bronchial biopsy, electron microscopy, ciliary visualisation and genetic testing (data available from the authors).

\section{Screening for $P C D$}

Nasal nitric oxide (NO), recommended for screening by the task force, was used by $46 \%$ of centres, varying from $29 \%$ in eastern Europe to $68 \%$ in northern countries (table 3). Variants of the saccharine test, considered as unreliable in children, 


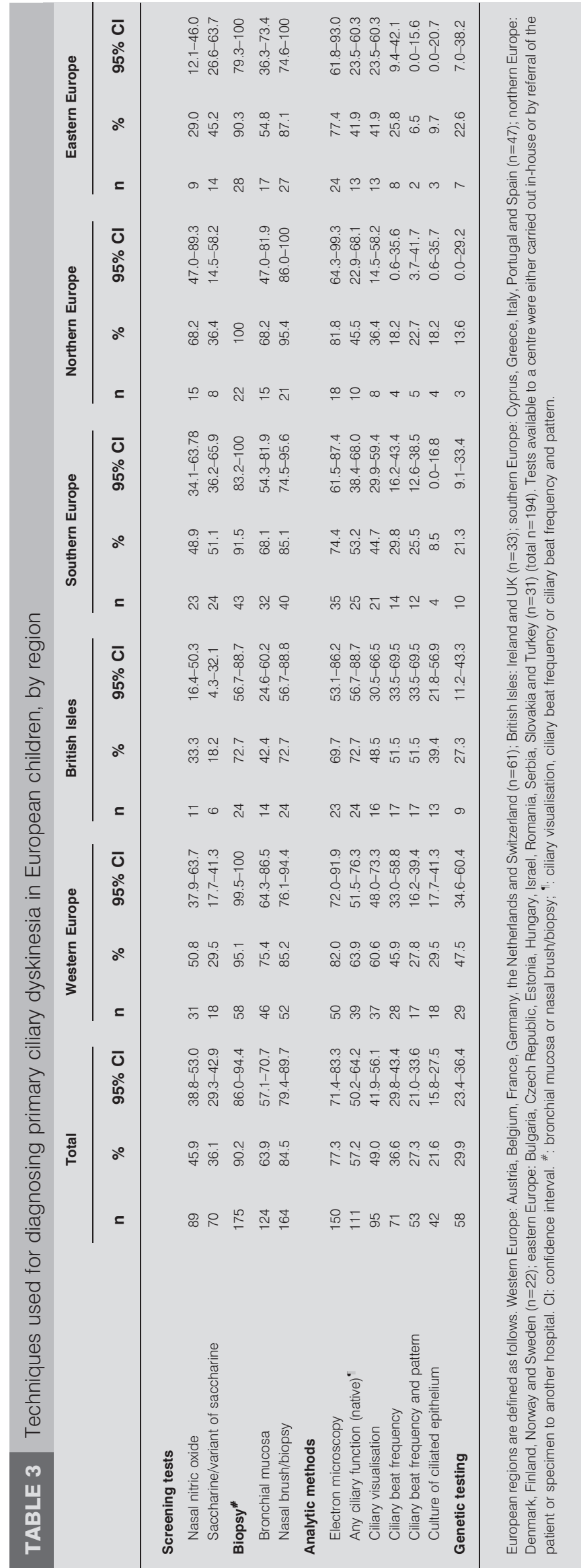

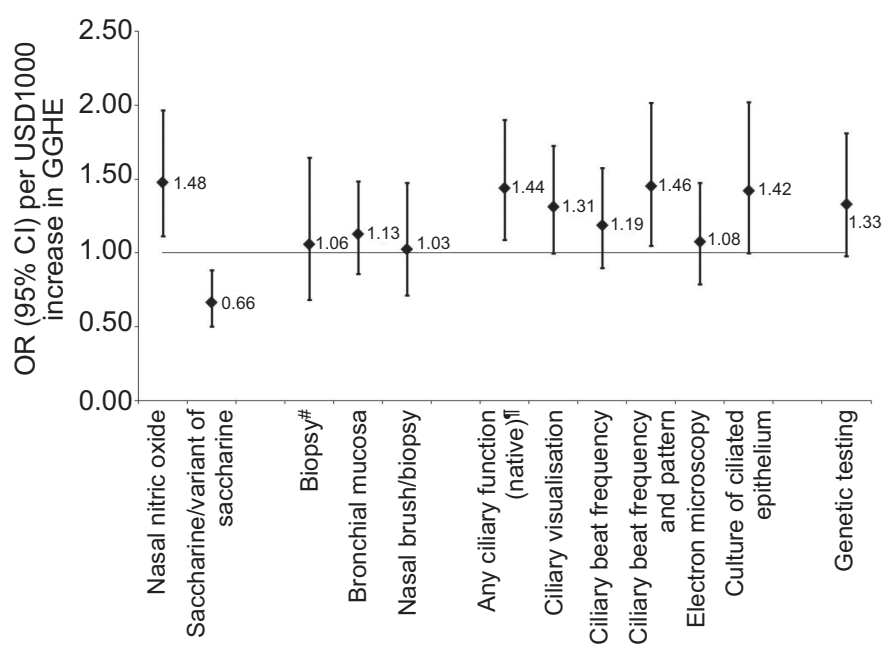

FIGURE 1. Association between diagnostic tests used to diagnose primary ciliary dyskinesia in children and the general government expenditure on health (GGHE) in the country (unadjusted results; $\mathrm{n}=26$ ). OR: odds ratio; Cl: confidence interval. \#: bronchial mucosa or nasal brush/biopsy; ": ciliary visualisation, ciliary beat frequency or ciliary beat frequency and pattern.

were used by $36 \%$ of centres $(18 \%$ in the British Isles and $51 \%$ in southern Europe).

\section{Confirmation of the diagnosis}

Nearly all centres (90\%) used a bronchial (64\%) or nasal (85\%) biopsy specimen of ciliated cells, as recommended (table 3). Electron microscopy, however, was only available to $77 \%$ of centres and native ciliary function tests to $57 \%$ of centres. Many could only assess ciliary beat frequency, with only $27 \%$ of centres being able to measure both beat frequency and pattern, varying from $7 \%$ in eastern Europe to $52 \%$ in the British Isles. Worryingly, $16 \%$ of all centres used neither electron microscopy nor ciliary function tests. Culture of ciliated epithelium and genetic analyses were only available to few centres, again with important regional differences.

\section{Determinants of use of different diagnostic techniques}

Higher GGHE (fig. 1) was associated with an increased likelihood to use nasal NO (OR 1.48 per USD1,000 increase in GGHE) and a decreased likelihood to use saccharine tests for screening (OR 0.66). Similarly, GGHE was associated with more access to native ciliary function tests (OR 1.44), culture of ciliated epithelium (OR 1.42) and genetic testing (OR 1.33).

In a multivariable regression adjusting simultaneously for size of centre and region (table 4), regional differences and number of PCD patients treated in the centre remained significantly associated with use of diagnostic methods. The number of patients treated per centre was a stronger determinant of diagnostic techniques than the type of centre (tertiary or smaller) or the number of specialists in the PCD team. Results for region remained similar, when we adjusted the models for type of centre rather than for number of treated patients.

\section{Treatment of PCD}

A large number of treatments were used either routinely for all patients, or sometimes for specific patients (table E3 of the 


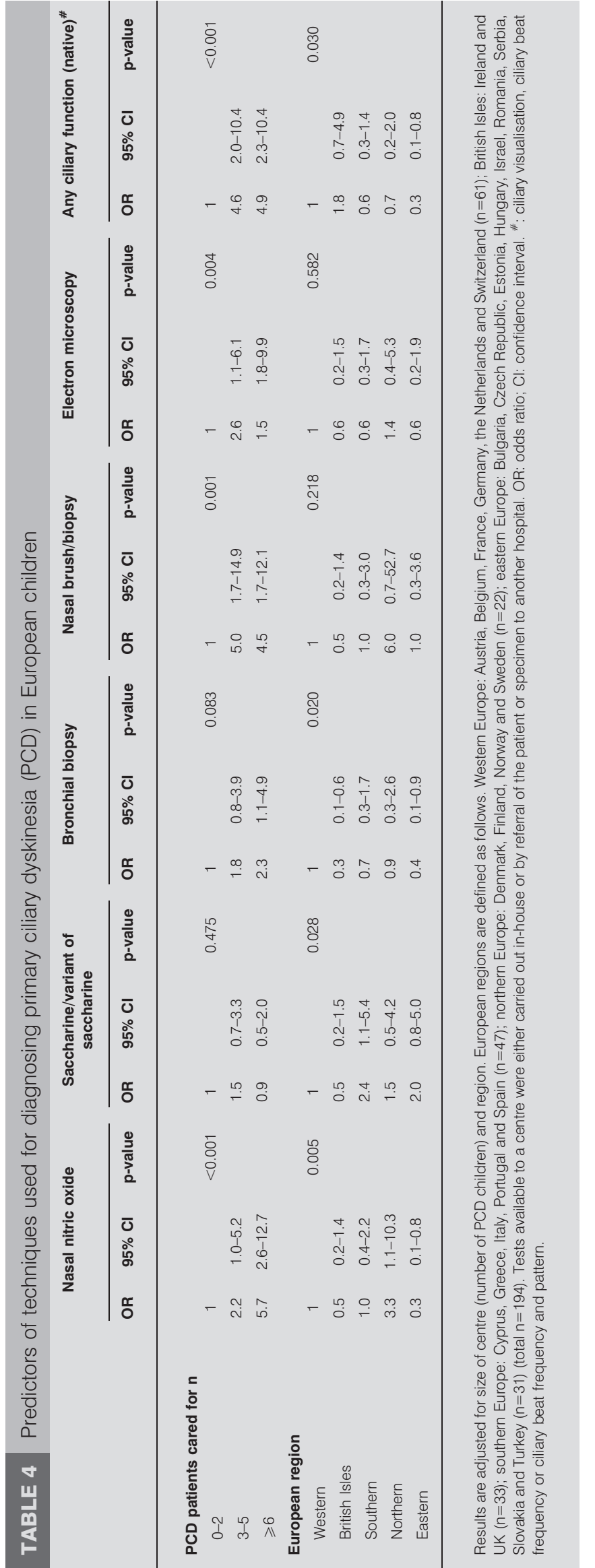

- Routinely (for all children) $\quad \square$ Frequently or sometimes $\square$ Never (for some children)
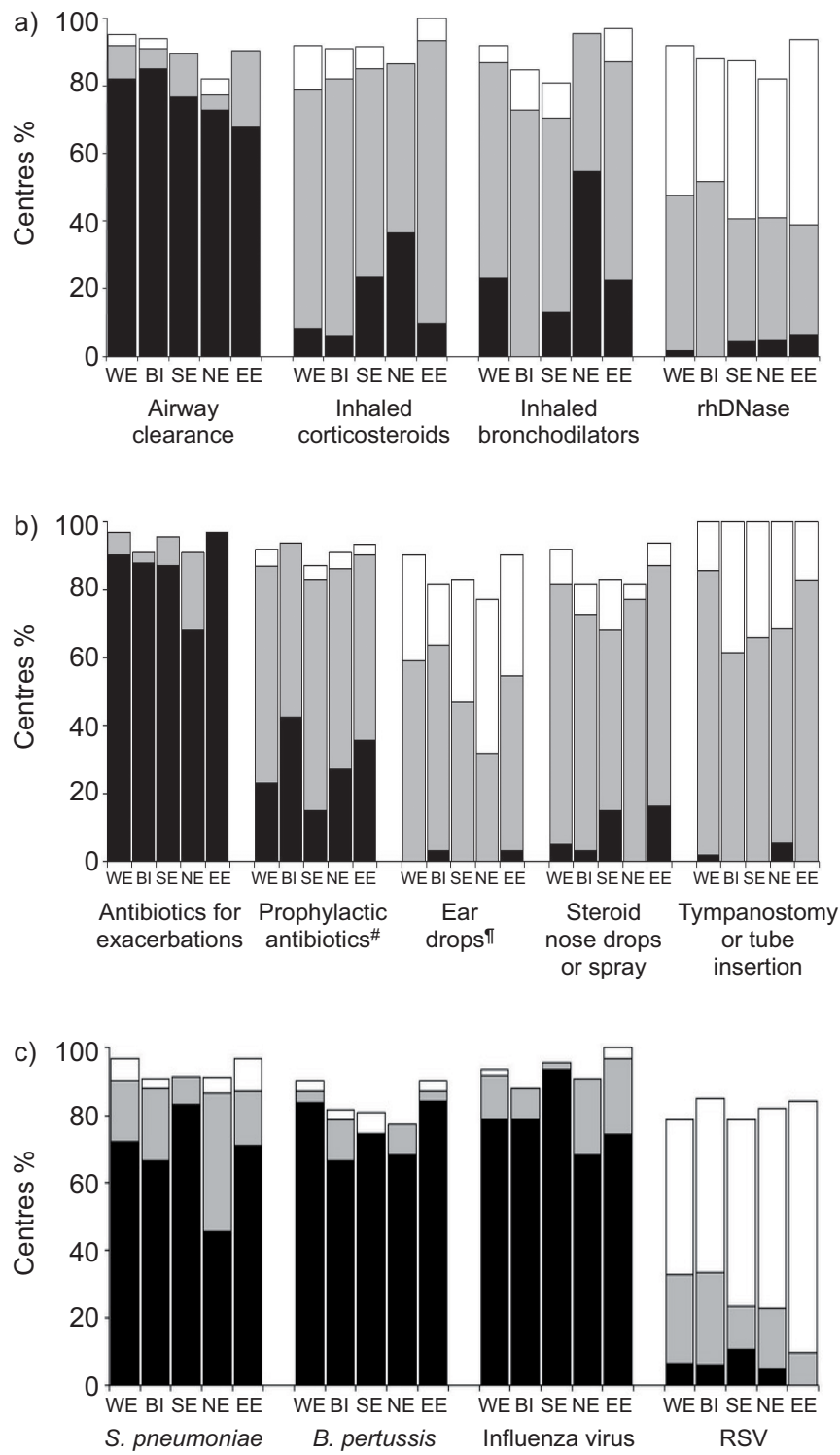

FIGURE 2. Treatment of primary ciliary dyskinesia patients in 26 countries, comparing European regions: a) airway clearance and inhalants; b) antibiotics and ear, nose and throat treatments; and c) immunisations. Results are based on the 194 centres reporting patients as follows. Western Europe (WE): Austria, Belgium, France, Germany, the Netherlands and Switzerland ( $n=61)$; British Isles $(\mathrm{BI})$ : Ireland and UK $(\mathrm{n}=33)$; southern Europe (SE): Cyprus, Greece, Italy, Portugal and Spain $(n=47)$; northern Europe (NE): Denmark, Finland, Norway and Sweden ( $n=22)$; eastern Europe (EE): Bulgaria, Czech Republic, Estonia, Hungary, Israel, Romania, Serbia, Slovakia and Turkey $(n=31) .{ }^{*}$ : prophylactic nebulised antibiotics, prophylactic oral antibiotics, intermittent oral antibiotics, regular intravenous antibiotics or intermittent intravenous antibiotics; " ": quinolone or aminoglycoside eardrops. rhDNase: recombinant human deoxyribonuclease; S. pneumoniae: Streptococcus pneumoniae; B. pertussis: Bordetella pertussis; RSV: respiratory syncytial virus. 
TABLE 5 Predictors of treatments of primary ciliary dyskinesia (PCD) in European children: airway clearance therapy, inhaled corticosteroids, recombinant human deoxyribonuclease (rhDNase) and antibiotics

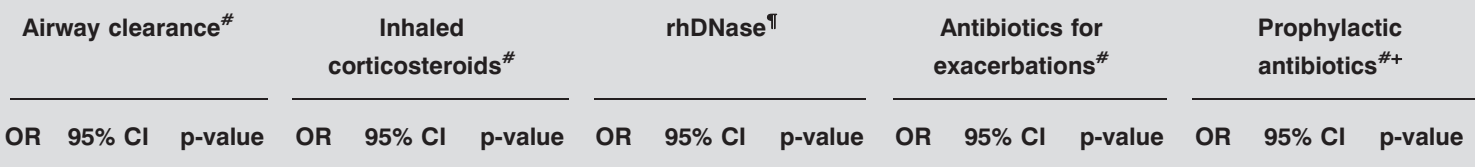

\begin{tabular}{|c|c|c|c|c|c|c|c|c|c|c|c|c|c|c|c|}
\hline $3-5$ & 1.7 & $0.7-4.0$ & & 0.4 & $0.1-1.2$ & & 1.6 & $0.7-3.6$ & & 2.8 & $0.8-9.5$ & & 1.9 & $0.8-4.5$ & \\
\hline$\geqslant 6$ & 3.2 & $1.3-7.7$ & & 0.3 & $0.1-0.9$ & & 2.6 & $1.3-5.4$ & & 2.7 & $0.9-7.8$ & & 1.3 & $0.6-2.9$ & \\
\hline \multicolumn{16}{|c|}{ European region } \\
\hline Western & 1 & & 0.302 & 1.00 & & 0.063 & 1.00 & & 0.326 & 1.00 & & 0.215 & 1.00 & & 0.063 \\
\hline British Isles & 1.4 & $0.4-4.5$ & & 0.7 & $0.1-3.6$ & & 1.4 & $0.6-3.3$ & & 0.8 & $0.2-3.3$ & & 2.5 & $01.0-6.3$ & \\
\hline Eastern & 0.4 & $0.1-1.1$ & & 1.4 & $0.3-6.4$ & & 0.5 & $0.2-1.2$ & & 2.9 & $0.3-26.0$ & & 1.8 & $0.7-4.78$ & \\
\hline
\end{tabular}

Results are adjusted for size of centre (number of PCD children) and region. European regions are defined as follows. Western Europe: Austria, Belgium, France, Germany, the Netherlands and Switzerland $(n=61)$; British Isles: Ireland and UK $(n=33)$; southern Europe: Cyprus, Greece, Italy, Portugal and Spain ( $n=47)$; northern Europe: Denmark, Finland, Norway and Sweden ( $n=22)$; eastern Europe: Bulgaria, Czech Republic, Estonia, Hungary, Israel, Romania, Serbia, Slovakia and Turkey $(n=31)$ (total $n=194)$. OR: odds ratio; $\mathrm{Cl}$ : confidence interval. ${ }^{*}$ : routinely used (for all children); ${ }^{\natural}$ : frequently or sometimes used (for some children); ${ }^{+}$: prophylactic nebulised antibiotics, prophylactic oral antibiotics, intermittent oral antibiotics, regular intravenous antibiotics or intermittent intravenous antibiotics.

online supplement) (fig. 2). In total, $78 \%$ of centres prescribed an airway clearance therapy and $28 \%$ a formal exercise programme to all patients (fig. 2a). Most centres prescribed inhaled bronchodilators and corticosteroids (ICS) only to selected patients. However, $20 \%$ of centres prescribed bronchodilators routinely to all patients and $15 \%$ did so for ICS. Recombinant human deoxyribonuclease (rhDNase) was prescribed (sometimes or routinely) by $45 \%$ of centres.

Antibiotics were used for treatment of exacerbations in $88 \%$ of centres (fig. 2b). Fewer prescribed prophylactic or intermittent antibiotics, either routinely (few centres) or sometimes (many). Tympanostomy or tube insertion was usually only applied to selected children. Immunisation against Bordetella pertussis, Streptococcus pneumoniae and influenza was part of routine management in $77 \%, 71 \%$ and $80 \%$ of centres respectively (fig. 2c).

Management varied widely between centres within a country and between regions within Europe (table E4 of the online supplement) (fig. 2). In northern European countries, PCD was often treated like asthma with routine use of inhaled steroids and $\beta$-agonists. Airway clearance, antibiotics for exacerbations and vaccinations, comparatively cheap therapies considered essential by experts, were used in the majority of centres. Still, an important minority did not prescribe them to all patients. In contrast, rhDNase, an exceedingly expensive treatment, was used by nearly $50 \%$ of centres, despite a total lack of evidence for its effectiveness in PCD.

\section{Determinants of use of different treatments}

We examined the factors that were associated with different treatment modes, with a focus on routine use of airway clearance and prophylactic antibiotics (two measures recommended for all patients in the consensus statement), and on routine use of ICS and rhDNase (two measures not recommended for all patients). In univariable analysis, airway clearance therapy and rhDNase were prescribed less often in centres with fewer patients and in eastern Europe. In contrast, ICS were used more often in smaller centres and in southern and northern Europe, compared with western and eastern Europe and the British Isles. Antibiotic treatment did not vary by region. Multivariable analysis (table 5) confirmed these findings. Again, the number of PCD patients cared for in the centre was a stronger explanatory factor for treatments than size of the PCD team or type of centre (tertiary or smaller). Adjusting the models for type of centre rather than for number of treated patients did not change the results for regions. For the frequency of use of rhDNase, we did not find any correlations between the number of cystic fibrosis patients cared for in the centre and the number of PCD patients.

\section{Follow-up care}

Frequency of follow-up visits in centres varied between every 3-4 months (134 centres, 69\%), every 6 months (40 centres, $21 \%$ ), yearly (seven centres, $4 \%$ ) and "at parents' request" (four centres, $2 \%$ ). This did not differ significantly by type of centre ( $p=0.429$; table E5 of the online supplement) and there were small differences between the western region compared to the other European regions $(\mathrm{p}=0.005$; table E6 of the online supplement).

The PCD team included a respiratory physician (180 centres, $93 \%)$, a physiotherapist $(165,85 \%)$, an ear, nose and throat (ENT) surgeon (136, 70\%), an audiologist (114,59\%), a specialist nurse $(94,49 \%)$, a social worker $(89,46 \%)$, a psychologist $(89$, $46 \%$ ) and a radiologist $(128,66 \%)$. Audiologists, radiologists and ENT surgeons were more often part of the PCD team in tertiary care centres than in smaller hospitals $(p=0.003, p=0.001$ and $\mathrm{p}<0.001$, respectively; table E7 of the online supplement) and 
there were small differences between regions, particularly for radiologists, social workers and psychologists $(p=0.211$, $\mathrm{p}=0.656$ and $\mathrm{p}=0.288$, respectively; table $\mathrm{E} 8$ of the online supplement).

\section{DISCUSSION}

This is the first study to describe diagnostic work-up and management of PCD in European children and to compare it with current expert recommendations. Until recently, there were no international guidelines for management of PCD in children, only recommendations from the UK [1, 5, 11-15]. This survey, describing the situation before publication of the ERS Task Force guidelines [7], found that PCD care is decentralised, with an average number of only four patients per centre, and that diagnostic work-up and management is heterogeneous and often contrasts international recommendations.

\section{Strengths and limitations}

Although most countries had a response rate of $>66 \%$, response rates varied between countries and only paediatric centres were approached. Moreover, because of national differences in healthcare systems, some countries collected data from tertiary, secondary and primary care, while in countries with highly centralised PCD care, only tertiary centres were approached. This could have introduced a bias, but the alternative, excluding all data not from tertiary care, would be open to the same criticism. Furthermore, the definition of primary, secondary and tertiary varies across Europe, so even combining so-called "tertiary" centres would not result in a homogeneous study group. Therefore, our point estimates (e.g. the proportion of centres using a specific management) might not be totally representative for Europe. Indeed, the true variability in diagnostic approaches and treatments is likely to be even larger than suggested by our study, because centres that did not participate or are led by non-paediatric physicians are likely to have different management strategies.

Our data are derived from questionnaires to physicians and not from chart reviews, and therefore represent the physicians' knowledge and intentions rather than what is actually done. Some respondents might have been unaware of diagnostic techniques available in their own country. In England, for instance, since 2006, all patients can be referred to nationally funded PCD diagnostic centres, where nasal NO, ciliary visualisation, beat function, electron microscopy and cell culture are available. Clearly, not all respondents were aware of the repertoire of diagnostics following referral to the national service.

\section{Organisation of care}

Recommendations state that patients with PCD should be seen for full or shared care in a centre specialising in the condition (table 1) [7]. Only three countries had centralised care in one national reference centre. Everywhere else, PCD care was split among many tertiary and secondary care centres, with only four patients per average centre. Considering that the response rate to the survey was not $100 \%$, the number of centres involved in PCD care must be even higher and the median number of patients per centre lower.

\section{Diagnosis of PCD}

Nasal NO measurements, a helpful tool in PCD screening in all age groups [16] are only available to $48 \%$ of tertiary care and $37 \%$ of smaller centres. Conversely, the saccharine test and its variants, which are unreliable in young children, are still widely used, particularly in low-resource countries. Reassuringly, 90\% of centres based their final diagnosis on samples of ciliated epithelium obtained via nasal or bronchial brushing or biopsies. The remaining $10 \%$ of centres were, however, not able to perform ciliary analysis or refer children to a centre offering this. Nasal brushing represents an elegant, simple, well-tolerated and only minimally invasive way to collect samples of ciliated epithelium. Despite that, bronchial biopsies were more commonly used, perhaps because bronchoscopy allows several differential diagnoses for a chronic bronchitis (foreign body aspiration, airway anomalies or recurrent infections) to be ruled out.

The way these samples were further analysed differed considerably between countries. This might be for historical reasons (techniques being used preferentially in places where they were developed) and financial reasons (more sophisticated methods being unavailable in low-resource countries). Electron microscopy for analysis of structural defects was available in most centres. In contrast, only a few places performed functional tests such as ciliary beat pattern analysis, which are essential for children with normal electron microscopy, as some patients with PCD have normal ciliary ultrastructure [17, 18]. The same applies to genetic testing, culture of ciliated epithelium or immunofluorescence, but in contrast to beat pattern analysis, these investigations are only useful in a minority of patients. Functional tests were rarely performed in small centres and resource-poor eastern European countries.

This survey assessed accessibility to diagnostic tests but not quality of measurements. It seems reasonable that specialist investigations should only be available at centres with a high throughput of PCD diagnostic samples to ensure quality of assessments.

\section{Treatment of PCD}

We found a wide range of different therapies in use for PCD patients. The use of treatments developed for cystic fibrosis patients, such as airway clearance techniques, regular inhaled antibiotics, rhDNase or hypertonic saline, demonstrates that many paediatricians in Europe observe similarities between these diseases and extrapolate evidence from cystic fibrosis clinical trials. However, the pathophysiology of the two diseases is different and the evidence is lacking that treatments that are beneficial in cystic fibrosis have any value in PCD [19].

There were considerable within- and between-country differences. For instance, rhDNase was more widely used in centres treating more PCD patients. Although these centres usually treated more cystic fibrosis patients, we found no association between number of cystic fibrosis patients per centre and use of rhDNase for PCD patients in the centre. In southern, northern and eastern Europe, but not in western Europe or Britain, ICS were often used for treatment of PCD, although evidence for their effectiveness is lacking.

Importantly, the two least disputed elements of PCD treatment, airway clearance therapy and immediate use of 
high-dose oral antibiotics for every exacerbation, were used by the large majority of centres. Still, a significant minority (10$15 \%)$ did not endorse these recommendations. The fact that these cheap and effective treatments were sometimes neglected in centres where expensive drugs such rhDNase were prescribed suggests the importance of the ERS Task Force in trying to standardise management in PCD patients.

\section{Follow-up care}

Although in our survey $90 \%$ of all centres reported planning follow-up visits for PCD patients every 3-6 months, it is uncertain whether that actually happens, especially for adolescents. Reasons might include poor compliance at teen age or different management strategies of adult chest physicians treating this age group. This is an area of concern because lung function deteriorates in young adults [2, 20, 21], so treatment should be continued or even intensified in adolescence.

\section{Implications and conclusions}

In summary, this large survey assessing management of paediatric PCD patients in Europe showed a wide variety of diagnostic practices and treatments, and only partial alignment with the recommendations that were published shortly after this survey [7]. This mirrors the lack of evidence on the effectiveness of treatments, the inexistence (until recently) of international guidelines and the lack of resources to implement current knowledge in some regions. In most countries, care for PCD patients was split among a large number of centres, and there is evidence that this affected quality of diagnostics and care.

The following issues might profitably be addressed at European Union level. First, there is a need to centralise management to one or few reference centres per country with specialised techniques. Secondly, resource implications prevent proper PCD diagnosis in many parts of Europe. It is likely that while many patients remain undiagnosed, others are incorrectly labelled as having PCD. Thirdly, medications are used unlicensed, off-label and in a non-evidence based manner. This means that some children miss beneficial therapies while others are exposed to treatments that are potentially dangerous, or useless but costly.

Conducting this survey has resulted in a number of benefits. The network of medical institutions and the database of PCD patients can be used for planning randomised controlled trials in this rare disease and for collecting information on clinical aspects and long-term prognosis. An international registry of PCD patients with data on diagnosis, treatments and long-term follow-up would be the logical step from this study and an ideal instrument for further research on this rare disease. Once the evidence base for treatment of PCD is stronger, management recommendations can be updated and widely implemented in Europe.

\section{SUPPORT STATEMENT}

The European Respiratory Society has financially supported meetings of the Primary Ciliary Dyskinesia Task Force. This paper is not considered part of the official task force report; the full, official report has previously been published in the European Respiratory Journal (Eur Respir J 2009; 34: 1264-1276). C.E. Kuehni and M-P.F. Strippoli were funded by the Swiss National Science Foundation (PROSPER grants 3233-069348 and 3200-069349, SNF 3200B0-122341). Local data collection in the Czech Republic was supported by a research project of the
Ministry of Health (Czech Republic; no. MZ0FNM2005). This work was supported by the Ministry of Science, Serbia (grant no. 41004).

\section{STATEMENT OF INTEREST}

None declared.

\section{ACKNOWLEDGEMENTS}

Author affiliations are as follows. M-P.F. Strippoli, E. Maurer and C.E. Kuehni: Institute of Social and Preventive Medicine, University of Bern, Bern, Switzerland; T. Frischer: Universitätsklinik für Kinder- und Jugendheilkunde, Vienna, Austria; A. Barbato and D. Snijders: Dept of Paediatrics, University of Padua, Padua, Italy; J.S.A. Lucas: School of Medicine, Division of Infection, Inflammation and Immunity, NIHR Respiratory Research Unit, University of Southampton, Southampton, UK; E. Eber: Dept of Paediatrics and Adolescence Medicine, Medical University of Graz, Graz, Austria; B. Karadag: Division of Paediatric Pulmonology, Marmara University, Istanbul, Turkey; P. Pohunek: Division of Respiratory Diseases, Paediatric Dept, University Hospital Motol, Prague, Czech Republic; Z. Zivkovic: Children's Hospital for Lung Diseases and TB, Medical Center Dr Dragisa Misovic, and US Medical School, European University, Belgrade, Serbia; A. Escribano: Paediatric Pulmonology Unit, University Clinic Hospital, University of Valencia, Valencia, Spain; C. O'Callaghan: Portex Unit, Institute of Child Health, University College London, and Great Ormond Street Hospital, London, UK; A. Bush: Dept of Paediatric Respiratory Medicine, Royal Brompton Hospital, London, UK.

The members of the European Respiratory Society Task Force on primary ciliary dyskinesia in children are as follows. I. Azevedo: Dept of Paediatrics, Hospital São João, Porto, Portugal; G. Baktai: Paediatric Institute Svabhegy, Budapest, Hungary; A. Barbato: Dept of Paediatrics, University of Padua, Padua, Italy; J. De Blic: Dept of Pneumo-Allergology, Necker-Enfants Malades Hospital, Paris, France; A. Bush: Royal Brompton Hospital, London, UK; K-H. Carlsen: Voksentoppen BKL, Rikshospitalet University Hospital, Oslo, Norway; L. Larisa Dracea: Sectia Afectiuni Resporatorii, Spital de Copii, Brasov, Romania; E. Eber: Dept of Paediatrics and Adolescence Medicine, Medical University of Graz, Graz, Austria; S. Elborn: Respiratory Medicine Group, Centre for Infection and Immunity, Queen's University of Belfast, Belfast, UK; A. Escribano: Paediatric Pulmonology Unit, University Clinic Hospital, University of Valencia, Valencia, Spain; T. Frischer: Universitätsklinik für Kinder- und Jugendheikunde, Vienna, Austria; E. Haarman: Dept of Paediatrics and Neonatology, Emma Children's Hospital Academic Medical Centre, Amsterdam, the Netherlands; B. Hesselmar: Dept of Paediatrics, Institute of Clinical Sciences, Sahlgrenska Academy of Gothenburg University, Gothenburg, Sweden; C. Hogg: Royal Brompton Hospital, London, UK; M. Jorissen: Ear, Nose and Throat Dept, Head and Neck Surgery, University Hospitals Leuven, Leuven, Belgium; B. Karadag: Division of Paediatric Pulmonology, Marmara University, Istanbul, Turkey; C.E. Kuehni: Insitute of Social and Preventive Medicine (ISPM), University of Bern, Bern, Switzerland; M. Korppi: Paediatric Research Centre, Tampere University and University Hospital, Tampere University, Tampere, Finland; J.S. Lucas: School of Medicine, Division of Infection, Inflammation and Immunity, NIHR Respiratory Research Unit, University of Southampton, Southampton, UK; E. Maurer: Institute of Social and Preventive Medicine (ISPM), University of Bern, Bern, Switzerland; P. Minic: Mother and Child Health Institute of Serbia "Dr Vukan Cupic", Belgrade, Serbia and Montenegro; H. Mussaffi: Cystic Fibrosis Service, Pulmonary Institute, Schneider Children's Medical Centre of Israel, Petah-Tikva, Israel; K.G. Nielsen: Danish Paediatric Pulmonary Service, Rigshospitalet, Copenhhagen, Denmark; C. O'Callaghan: Portex Unit, Institute of Child Health, University College London and Great Ormond Street Hospital, London, UK; H. Omran: Klinik und Poliklinik für Allgemeindepaediatrie, University Hospital, Münster, Germany; P. Pohunek: Dept of Paediatrics, University Hospital 
Motol and 2nd School of Medicine, Charles University, Prague, Czech Republic; K. Priftis: Dept of Allergy-Pneumonology, Penteli Children's Hospital, Palea Penteli, Greece; M.A. Riikjärv: Tallinn Children's Hospital, Tallinn, Estonia; G. Petrova Stoyanova: Paediatric Clinic, Sofia, Bulgaria; V. Pohanka: Srobars Institute for TB and Respiratory Disease, Dolný Smokovec, Slovakia; D. Snijders: Dept of Paediatrics, University of Padua, Padua, Italy; M.P.F. Strippoli: Institute of Social and Preventive Medicine (ISPM), University of Bern, Bern, Switzerland; T. Svobodová: Dept of Paediatrics, University Hospital Motol, Prague, Czech Republic; P. Yiallouros: Cyprus International Institute for Environmental and Public Health, Cyprus University of Technology, Limassol, Cyprus; Z. Zivkovic: Children's Hospital for Lung Diseases and TB, Medical Center Dr Dragisa Misovic, Belgrade, and US Medical School, European University, Belgrade, Serbia.

The authors and all the members of the European Respiratory Society Task Force would like to thank all centres participating in the primary ciliary dyskinesia survey. Austria: Kardinal Schwarzenberg'sches Krankenhaus (Schwarzach), SMZ Ost Donauspital (Vienna), University Childrens Hospital (Graz), St Anna Kinderspital (Vienna), a.ö. Landeskrankenhaus (St Pölten), Klinikum Kreuzschwestern (Wels), Wilhelminenspital (Vienna), University Childrens Hospital (Vienna), LKH (Feldkirch), Medical University (Innsbruck), a.ö. LKH des Landes Kärnten (Klagenfurt), Landeskinder- und Frauenklinik (Linz), Paracelsus Medizinische Privatuniversität - Childrens Hospital (Salzburg); Belgium: Virge Jesse Ziekenhui (Hasselt), UZ (Leuven), UCL Mont-Godinne (Yvoir), Clinique Universitaire Saint-Luc (Brussels), AZ-VUB Jette (Brussels), UZ (Ghent), CHC St-Vincent (Rocourt), AT Middelheim Kon. Paola Kinderziekenhuis (Antwerp); British Isles: Our Ladys Childrens Hospital (Dublin, Ireland), St James University Hospital (Leeds, UK), Stirling Royal Infirmary (Fife, UK), Royal Devon and Exeter Foundations NHS Trust (Devon, UK), Royal Aberdeen Children's Hospital (Aberdeen, UK), Royal Brompton Hospital (London, UK), Addenbrookes NHS Trust (Cambridge, UK), Freeman Hospital (Newcastle Upon Tyne, UK), Royal Infirmary (Leicester, UK), Ninewells Hospital (Dundee, UK), Hospital (Nottingham, UK), Royal Blackburn Hospital (Blackburn, UK), Swansea NHS Trust (Swansea, UK), Kings Mill Hospital (Sutton in Ashfield, UK), Goodhope Hospital (Sutton Coldfield, UK), Glan Clwyd Hospital (Rhyl, UK), Southampton University Hospital NHS Trust (Southampton, UK), Royal Belfast Hospital Sick Children (Belfast, UK), Huddersfield Royal Infirmary (Huddersfield, UK), West Wales Hospital (Carmather, UK), Teaching Hospitals Foundation Trust (Bradford, UK), Children's Hospital for Wales (Cardiff, UK), York Foundation NHS Trust (York, UK), Royal Hospital for Sick Children (Edinburgh, UK), Children's Hospital (Bristol, UK), Shrewsbury and Telford Hospital NHS Trust (Shrewsbury, UK), Children's Hospital (Birmingham, UK), Children's University Hospital NHS Trust (Manchester, UK), Royal Cornwall Hospital Trust (Truro, UK), Derriford Hospital (Plymouth, UK), Children's Hospital (Oxford, UK), Royal Victoria Infirmary (Newcastle Upon Tyne), Royal Alexandra Children's Hospital (Brighton, UK), University Hospital (Cork, Ireland), South Devon Healthcare Trust (Devon); Bulgaria: University Hospital Alexendrovska (Sofia), University Hospital (Pleven); Cyprus: General Hospital (Larnaca), Archbishop Makarios Hospital (Nicosia), New Famagusta General Hospital (ParalimniFamagusta), General Hospital (Paphos), General Hospital (Limassol); Czech Republic: Teaching Hospital of Palacky University (Olomouc), University Hospital Motol (Prague); Denmark: University Hospital, Paediatric Pulmonary Service and Ciliary Lab (Copenhagen); Estonia: Children's Hospital (Tallinn), University Children's Clinic (Tartu); Finland: University Hospital (Tampere), University Hospital (Oulu), University Hospital (Kuopio), University Hospital (Helsinki); France: Armand Trousseau Hospital (Paris), Hôpital Necker Enfants Malades (Paris), Centre Hospitalier Intercommunal (Créteil), Hôpital des Enfants CHU (Toulouse), Hôpital Debrousse (Lyon), Hôpital Jeanne de Flandres (Lille); Germany: Kinder- und Jugendklinik (Erlangen),
University Children's Hospital Charite (Berlin), University Hospital (Freiburg), Universitätsklinik für Kinder- und Jugendmedizin (Tübingen), Praxis für Kinderpneumologie und Allergologie (Mannheim), Klinikum des Universität (Munich), Kinderpneumologie (Eckental), Universitätsklinik (Düsseldorf), Akad. Lehrkrankenhaus der J. Gutenberg-Universität Mainz (Worms), Evangelisches Krankenhaus (Bielefeld), Marien-Hospital (Wesel), Klinik für Kinder- und Jugendmedizin (Eberswalde), Klinikum (Augsburg), Kinderarztpraxis Laurensberg (Aachen), Universitätsklinikum Schleswig Holstein Campus (Kiel), Universitäts-Kinderklinik (Bochum), University (Cologne), Josefinum (Augsburg), Altonaer Kinderkrankenhaus (Hamburg); Greece: Athens Medical Centre (Athens), Penteli Children's Hospital (Athens), University Hospital (Patra), University (Thessaloniki), University Hospital of Crete (Heraklion); Hungary: Paediatric Insitute-Svabhegy (Budapest); Israel: Ziv Medical Centre (Sefad), Dana Children Hospital, Tel-Aviv Medical Centre (Tel-Aviv), Safra Children's Hospital, Sheba Medical Centre (Ramat-Gan), Shaare Zedek Medical Centre (Jerusalem), Rambam Medical Centre (Haifa), Hadassah Mt Scopus University Medical Centre (Jerusalem), Soroka Medical Centre (Beer-Sheva), Schneider Children's Medical Centre of Israel (Petah-Tikva); Italy: Paediatric Department University Bari (Bari), Ospedale Infantile Burlo Garofolo - Clinica Pediatrica (Trieste), Ospedale Macedonio Melloni (Milan), Paediatric Unit-General Hospital (Bolzano), UO di Fibrosi Cistica-Clinica Pediatrica (Parma), Centro di Supporto per la Fibrosi Cistica (Brescia), Institute of Respiratory Diseases (Milan), Paediatric Department-Federico II University (Naples), Azienda di Rilievo Nazionale e di Alta Specializzazione-ARNAS (Palermo), Anna Meyer Children's Hospital (Florence), Azienda Ospedaliera "Fate Bene Fratelli" (Milan), Clinica Pediatrica Università di Catania (Catania), Paediatric Department University Udine (Udine), Instituto "G. Gaslini" (Genoa), Paediatric Department, University of Padua (Padua), Paediatric Department University Pisa (Pisa), Ospedale Paediatrico Bambino Gesù (Rome), Cystic Fibrosis Centre (Verona), Clinica Pediatrica (Ancona); the Netherlands: VU University Medical Centre (Amsterdam), University Medical Centre (Maastricht), Amphia Ziekenhuis (Breda), University Medical Centre (Utrecht), Radbout University Medical Centre (Nijmegen), Sofia Children's Hospital - Erasmus MC (Rotterdam); Norway: Voksentoppen (Oslo), Ullevål University Hospital (Oslo), St Olav's Hospital (Trondheim); Portugal: Hospital de Nossa Senhora do Rosário (Barreiro), Hospital Pediátrico (Coimbra), Hospital de S. João (Porto), Hospital de Santa Maria (Lisbon); Romania: University Children's Hospital (Brasov), Children's University Hospital Alfred Rusescu (Bucharest), Emergency Children's Hospital G. Alexandrescu (Bucharest); Serbia: Children's Hospital for Lung Diseases and TB, Medical Center Dr Dragisa Misovic (Belgrade), US Medical School, European University (Belgrade), Institute for Mother and Child Health Care (Belgrade); Spain: Hospital de Jerez (Jerez de la Frontera), Corporacio Sanitaria Parc Tauli (Sabadell-Barcelona), Hospital Infantil La Paz (Madrid), Hospital Clínico Universitario (Valencia), Hospital Universitario de Valme (Seville), Hospital Universitario Virgen de la Arrixaca (Murcia), Hospital Universitario 12 de Octubre (Madrid), Hospital Miguel Servet (Zaragoza), Hospital Materno Infantil Carlos Haya (Málaga), Hospital Universitario La Fe (Valencia), Hospital Universitario Virgen Macarena (Seville), Hospital del Mar (Barcelona), Hospital Infantil Universitario Niño Jesús (Madrid), Hospital Universitario Son Dureta (Palma de Mallorca), Hospital Severo Ochoa (Madrid), Hospital Universitario Nuestra Señora de Candelaria (Santa Cruz de Tenerife), Hospital Universitario de Canarias (La Laguna, Tenerife), Hospital Basurto (Bilbao), Hospital Universitario Virgen de Las Nieves (Granada), HCU "Juan Canalejo" (La Coruña), Complejo Hospitalario Universitario (Albacete), Hospital Gregorio Marañon (Madrid), Hospital Universitario Materno Infantil Vall d'Hebron (Barcelona), Hospital Donostia (San Sebastián); Slovakia: Comenius University (Bratislava); Sweden: Bamkliniken (Karlskrana), Karolinska University Hospital-Huddinge (Stockholm), Sjukhuset (Västerviks), Queen Silvia Children's Hospital (Gothenburg), University Hospital 
(Lund), Lapplands Bamklinik (Gällivare), Barnallergimot NSK (Kungsbacha), Central Sjukhusset (Skovde), University Hospital (Orebro), Vrinnevi Hospital (Norrköping), Hospital (Helsinborg), Barukliniken (Soffetea), Central Sjukhusset (Kristanstad), KEF (Linköping), Clinical Sciences (Malmö), Centrallasarettet (Västeras); Switzerland: University Children's Hospital (Bern), Hôpital régional (Sion), Hôpital du Chablais (Aigle), Ente Ospedaliero Cantonale (Ticino), Hôpital EHNV (Yverdon-LesBains), Hopital régional (Délémont), Dr Ruettimann (Aarau), Kantonsspital (Chur), Centre Hospitalier Universitaire Vaudois (Lausanne), Hôpital régional (La-Chaux-De-Fonds), University Hospital (Geneva), University Children's Hospital (Zürich), Children's Hospital (Aarau), University Children's Hospital (Basel), Children's Hospital (St Gallen), Alpine Children's Hospital (Davos), Children's Hospital (Lucerne), Kantonsspital (Münsterlingen); Turkey: Marmara University Faculty of Medicine (Istanbul), Izzet Baysal University Hospital (Bolu), Dokuz Eylul University Hospital (Izmir), Cukurova University Faculty of Medicine (Adana), KTÜ Technical University Medical Faculty (Trabzon), Uludag University Medical Faculty (Bursa), Gaziantep University Faculty of Medicine (Gaziantep), Behcet Uz Training Hospital (Izmir), Dicle University Faculty of Medicine (Diyarbakir), Hacettepe University Faculty of Medicine (Ankara), Mersin University Faculty of Medicine (Mersin), Celal Bayar University Hospital (Manisa), Akdeniz University Hospital (Antalya), Gazi University Hospital (Ankara).

\section{REFERENCES}

1 Bush A, Chodhari R, Collins N, et al. Primary ciliary dyskinesia: current state of the art. Arch Dis Child 2007; 92: 1136-1140.

2 Noone PG, Leigh MW, Sannuti A, et al. Primary ciliary dyskinesia: diagnostic and phenotypic features. Am J Respir Crit Care Med 2004; 169: 459-467.

3 Lucas JSA, Walker WT, Kuehni CE, et al. Primary ciliary dyskinesia. Eur Respir Monogr 2011; 54: 201-217.

4 Jain K, Padley SP, Goldstraw EJ, et al. Primary ciliary dyskinesia in the paediatric population: range and severity of radiological findings in a cohort of patients receiving tertiary care. Clin Radiol 2007; 62: 986-993.

5 O'Callaghan C, Chilvers M, Hogg C, et al. Diagnosing primary ciliary dyskinesia. Thorax 2007; 62: 656-657.

6 Bush A, Ferkol T. Movement: the emerging genetics of primary ciliary dyskinesia. Am J Respir Crit Care Med 2006; 174: 109-110.
7 Barbato A, Frischer T, Kuehni CE, et al. Primary ciliary dyskinesia: a consensus statement on diagnostic and treatment approaches in children. Eur Respir J 2009; 34: 1264-1276.

8 United Nations. Definition of Major Areas and Regions. 2005. http://esa.un.org/unpp/index.asp?panel=5. Date last updated: March 11, 2009. Date last accessed: February 6, 2009.

9 WHO. WHO Statistical Information System (WHOSIS). www. who.int/whosis/en/ Date last updated: February 6, 2009. Date last accessed: February 6, 2009.

10 Kuehni CE, Frischer T, Strippoli MP, et al. Factors influencing age at diagnosis of primary ciliary dyskinesia in European children. Eur Respir J 2010; 36: 1248-1258.

11 Bush A, O'Callaghan C. Primary ciliary dyskinesia. Arch Dis Child 2002; 87: 363-365.

12 Chilvers MA, Rutman A, O'Callaghan C. Ciliary beat pattern is associated with specific ultrastructural defects in primary ciliary dyskinesia. J Allergy Clin Immunol 2003; 112: 518-524.

13 Bush A. Primary ciliary dyskinesia. Acta Otorhinolaryngol Belg 2000; 54: 317-324.

14 Bush A, Cole P, Hariri M, et al. Primary ciliary dyskinesia: diagnosis and standards of care. Eur Respir J 1998; 12: 982-988.

15 Hadfield PJ, Rowe-Jones JM, Bush A, et al. Treatment of otitis media with effusion in children with primary ciliary dyskinesia. Clin Otolaryngol Allied Sci 1997; 22: 302-306.

16 Marthin JK, Nielsen KG. Choice of nasal nitric oxide technique as firstline test for primary ciliary dyskinesia. Eur Respir J 2011; 37: 559-565.

17 Bartoloni L, Blouin JL, Pan Y, et al. Mutations in the DNAH11 (axonemal heavy chain dynein type 11) gene cause one form of situs inversus totalis and most likely primary ciliary dyskinesia. Proc Natl Acad Sci USA 2002; 99: 10282-10286.

18 Schwabe GC, Hoffmann K, Loges NT, et al. Primary ciliary dyskinesia associated with normal axoneme ultrastructure is caused by DNAH11 mutations. Hum Mutat 2008; 29: 289-298.

19 Wills PJ, Wodehouse T, Corkery K, et al. Short-term recombinant human DNase in bronchiectasis. Effect on clinical state and in vitro sputum transportability. Am J Respir Crit Care Med 1996; 154: 413-417.

20 Hellinckx J, Demedts M, De Boeck K. Primary ciliary dyskinesia: evolution of pulmonary function. Eur J Pediatr 1998; 157: 422-426.

21 Marthin JK, Petersen N, Skovgaard LT, et al. Lung function in patients with primary ciliary dyskinesia: a cross-sectional and 3decade longitudinal study. Am J Respir Crit Care Med 2010; 181: 1262-1268. 